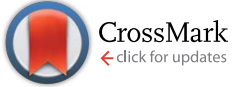

Cite this: J. Mater. Chem. A, 2016, 4 , 4490

Received 21st January 2016 Accepted 25th February 2016

DOI: $10.1039 / \mathrm{c} 6 \mathrm{ta} 01670 \mathrm{~g}$

www.rsc.org/MaterialsA

\title{
A re-evaluation of transparent conductor requirements for thin-film solar cells $\uparrow$
}

\begin{abstract}
Daniel A. Jacobs, ${ }^{\star}$ Kylie R. Catchpole, Fiona J. Beck and Thomas P. White
The needs of thin-film solar cells are a significant driver in transparent conductor research, making it vital that these requirements are properly understood. Here we demonstrate that the oft-quoted need for sheet resistances less than $10 \Omega \mathrm{sq}^{-1}$ arises only when the addition of a metal grid is unfeasible, and for cells of a particular size. In addition we show that the performance of a highly transparent layer with a metal grid is generally superior to that of a single layer fulfilling the $10 \Omega \mathrm{sq}^{-1}$ requirement without a grid. In order to clarify these issues we introduce simple measures of electrode performance which correspond directly to cell efficiency. These specialized figures of merit can be applied to electrodes with or without a metal grid, and also to those embedded in a tandem cell where good electrode performance is often imperative. By comparison we show that the ratio of DC to optical conductivity, the most widely used figure of merit for transparent conductors, is a bad predictor of performance in a solar cell. Our work jointly motivates the development of scalable techniques for incorporating thin metal wires into front-side electrodes, and research into transparent conductors which prioritize transparency over sheet resistance.
\end{abstract}

\section{Introduction}

Transparent conductors are a crucial enabling technology for the future of thin-film solar cells. Contenders for this role range from the familiar transparent conducting oxides such as indium- and fluorine-doped tin oxide, to graphene, carbon nanotubes, metallic nanowires, nano-patterned metallic films, conducting polymers and a variety of composites. ${ }^{1-3}$ Each technology reaches a different compromise between the two primary properties of any transparent conductor, that is its optical transparency and sheet resistance. Consequently, the required balance between transparency and sheet resistance can have a profound effect on which technologies and processes are declared relevant, and which receive the most attention.

The issue of transparent conductor (TC) requirements has been the subject of a renewed focus recently with the advent of perovskite cells, which have attractive characteristics for use in a tandem arrangement with either conventional silicon cells or other perovskites. ${ }^{\mathbf{4} 5}$ Tandem concepts such as the 4-terminal mechanical stack require as many as three transparent electrodes in total, putting a greater than usual pressure on electrode transparency. ${ }^{6}$ The 2 -terminal monolithically integrated tandem cell avoids the need for intermediate electrodes, but suffers losses of a different nature due to the necessity of

Centre for Sustainable Energy Systems, Research School of Engineering, The Australian National University, Canberra, Australian Capital Territory, Australia. E-mail: daniel. jacobs@anu.edu.au

$\dagger$ Electronic supplementary information (ESI) available. See DOI: $10.1039 /$ c6ta01670g current-matching between cells, making transparent electrode performance a key factor in the competition between these two designs. Finding a suitable transparent conductor for the rear of the perovskite top-cell is one of the major challenges that will need to be overcome before a viable 4-terminal tandem device can be demonstrated. ${ }^{7,8}$ In the absence of metallization, such a conductor should have a sheet resistance no greater than about $10 \Omega \mathrm{sq}^{-1},{ }^{9}$ a challenging requirement to meet at high transparency. With metallization in the form of a conducting grid the required sheet resistances are considerably relaxed, allowing for the use of thinner layers which block less of the valuable light.

Transparent conductors are usually among the first or the last layers to be deposited in a thin-film solar cell. Electrodes which are deposited last, as in the window layer of a CIGS cell ${ }^{10}$ or the rear electrode of a semitransparent perovskite cell, ${ }^{11}$ can be metallized with little difficulty. Electrodes which are deposited first will affect the topography of all subsequent layers, so that any wires should be kept below a threshold height depending on the device structure to avoid producing shunt pathways or other undesirable features. Alternatively, wires may be embedded into the front-surface substrate so as to produce a relatively flat surface for subsequent layers, for example, by using metallic paste to fill grooves fashioned with an imprint ${ }^{12-14}$ or laser scribing process. The wire sizes we consider lie in the range of 5-30 $\mu \mathrm{m}$, whose upper limit falls within the present capability of industrially-relevant techniques such as screen, ${ }^{15}$ ink-jet ${ }^{16,17}$ and flexographic printing. ${ }^{18,19}$ Smaller metal wires with widths $<20 \mu \mathrm{m}$ can be fabricated using a variety of 
techniques including nano-imprint lithography, ${ }^{20}$ microcontact printing $^{21}$ and nanotransfer printing, ${ }^{22}$ although it is beyond the scope of this paper to assess whether any of these techniques are viable candidates for low-cost mass-production in the near-future. Instead, we analyze the effects of metallization on transparent conductor requirements and performance, and in doing so provide impetus for the improvement of metallization technology.

We begin by reviewing the basic impact of ohmic and transparency loss on the efficiency of a solar cell. These loss mechanisms are tied together by introducing the efficiency factor, a measure of the TC's impact on its parent cell's efficiency, which we then use to perform a comparative study of TC technologies under different schemes of metallization. Finally, we give evidence that the standard figures of merit for transparent conductors may not be reliable indicators of performance in a solar cell, and suggest some simple alternatives based on the efficiency factor.

\section{Transparent conductor requirements with metallization}

In a homogeneous conducting layer the trade-off between sheet resistance and transparency is largely unavoidable, as increasing the planar carrier density for the sake of conductivity will tend to reduce transparency (one can circumvent this by increasing the carrier mobility instead, but this provides only a limited scope for improvement). However, without the constraint of homogeneity, there is much to be gained in transparency by condensing carriers into a sparse network of dense filaments. In the limit of high concentration such networks become effectively metallic and opaque, with a transparency determined only by their covering fraction, and can therefore be made almost arbitrarily tall without affecting transparency. For example, a layer of silver $50 \mathrm{~nm}$ thick has a transparency close to zero over the visible range, whereas the same volume of metal (having the same capacity to carry current over large distances) will produce an array of wires 1 $\mu \mathrm{m}$ tall with a covering fraction of just $5 \%$ or $95 \%$ transparency. Of course in heterogeneous conductors of this kind, such as arrays of regular or random metal wires, it becomes necessary to conduct current to the wires from all the intervening spaces, but as these distances are much smaller than the cell dimensions this task can be accomplished with only a very thin or lightly doped layer. This explains why the combination of a homogeneous layer to collect current, and a network of metallic wires to transport it over macroscopic distances, is so effective at achieving efficient carrier transport with high transparency. ${ }^{23-25}$ Different possibilities for incorporating metal wires into a typical thin-film cell structure are illustrated in Fig. 1(b and c).

Whether or not a transparent conductor is metallized, the optimal balance between its sheet resistance and transparency is determined by the joint effect of these properties on its cell's efficiency. To model this effect we will at first consider the top electrode on a stand-alone cell (Fig. 1(a and b)). To a good approximation $^{26}$ the sheet resistance of such an electrode affects the cell output voltage, whilst its transparency affects the current. Eqn (1a) given below is for the reduction in voltage $V_{\mathrm{mp}} /$ $V_{\mathrm{mp}}^{0}$ in a cell with an un-metallized or "bare" transparent conductor (Fig. 1(a)), normalized to its nominal value in an idealized cell without any ohmic loss. The second eqn (1b) for the same factor is also standard ${ }^{27}$ and applies instead to a layer with metal wires as in Fig. 1(b and d). It is derived by assuming that all the collected current flows directly through the TC to wires spaced at a period of $p$, before being conducted along the wires to the cell's contact, busbar or interconnect regions separated by a distance $W$ (see Fig. $1(\mathrm{~d}))$ :

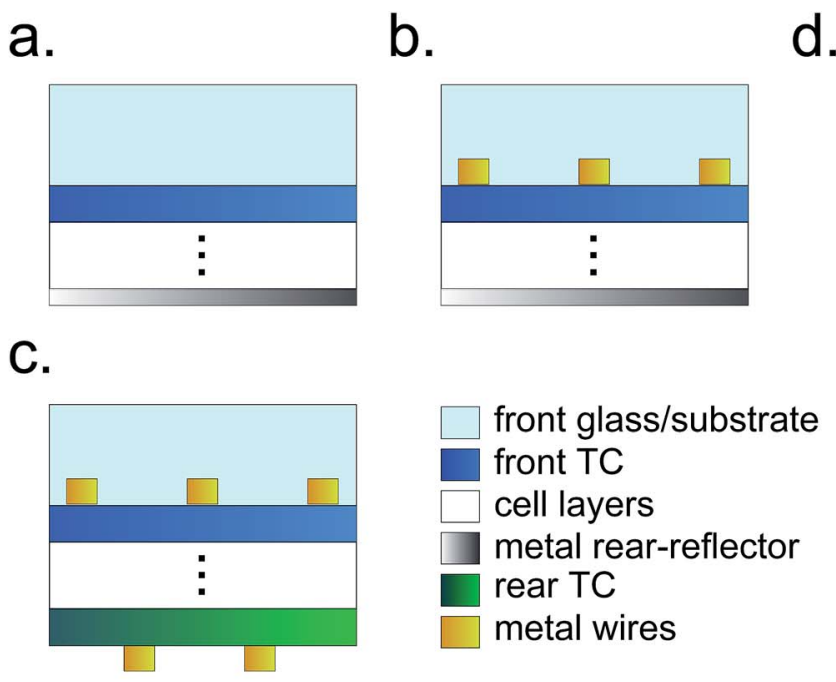

d.

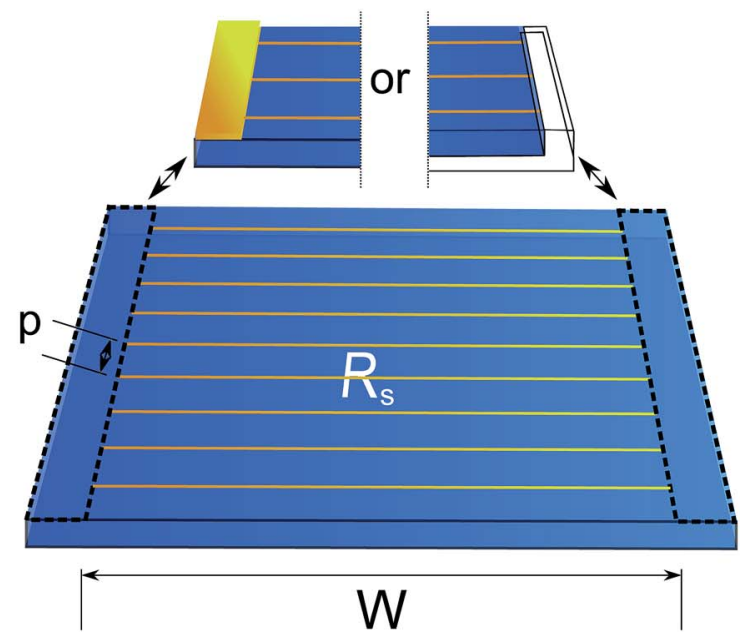

Fig. $1(a-c)$ Cross-sections of three basic cell types: (a) single cell with a bare front-side TC (b) single cell with metal wires inset into the front glass/substrate and (c) a semi-transparent cell with metallized front and rear TCs, such as might be used as the top-cell in a 4-terminal tandem device. (d) Schematic of a metallized TC in which the cell width $W$ is defined as the distance between bus-bars (standard Si-type module), the scribed interconnect regions (monolithic thin-film module) or cell contacts (lab cell). 


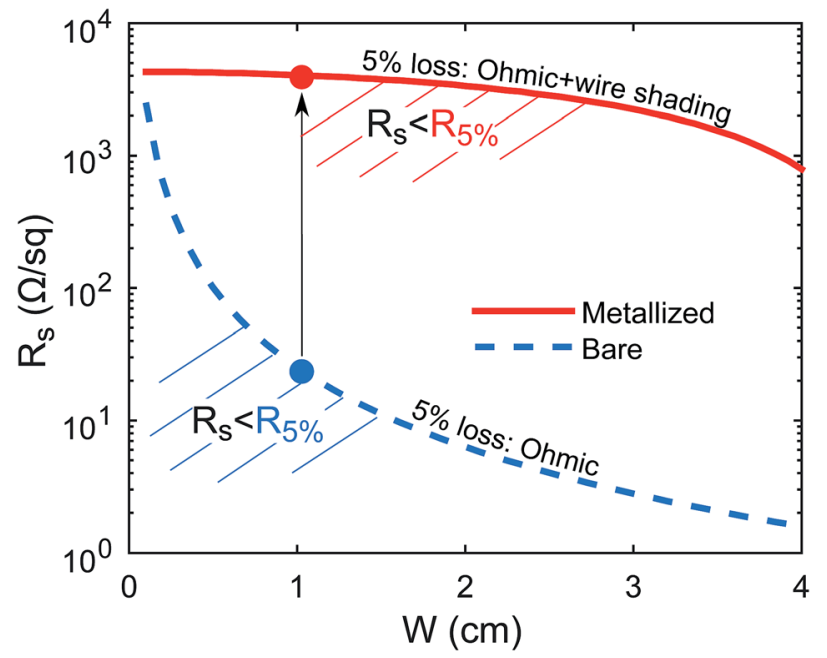

Fig. 2 Sheet resistance requirements as a function of the contact/ interconnect separation $W$ for a bare TC (dotted, blue) and metallized TC (solid, red) assuming $5 \%$ efficiency loss in a cell with $J_{\mathrm{mp}}^{0} / V_{\mathrm{mp}}^{0}=22$ $\mathrm{k}^{-1} \mathrm{~cm}^{-2}$. Losses were calculated assuming 100\% TC transparency, and therefore include only ohmic dissipation for the bare layer, and ohmic dissipation plus wire shading for the metallized layer.

$$
\frac{V_{\mathrm{mp}}}{V_{\mathrm{mp}}^{0}}=1-\frac{1}{3} \frac{J_{\mathrm{mp}}^{0} W^{2}}{V_{\mathrm{mp}}^{0}} \times\left\{\begin{array}{c}
R_{\mathrm{s}} \\
\frac{1}{4}(p / W)^{2} R_{\mathrm{s}}+R_{\mathrm{M}}
\end{array}\right.
$$

here $R_{\mathrm{M}}$ is the metallization sheet resistance given by $R_{\mathrm{M}}=\rho p /$ $w h, \rho$ is the metal resistivity (given the value $3 \times 10^{6} \Omega^{-1} \mathrm{~cm}$ hereafter), $w$ the wire width, $h$ the wire height, $J_{\mathrm{mp}}^{0}$ the collected current density assuming ideal transparency, and $R_{\mathrm{S}}$ is the bare TC sheet resistance. We note that in this expression the contribution of contact resistance between the TC and metal wires is neglected (see ESI $\dagger$ for justification). These expressions show that the effect of metallization is to replace the TC's sheet resistance $R_{\mathrm{s}}$ with an effective resistance $R_{\text {eff }}=\frac{1}{4}(p / W)^{2} R_{\mathrm{S}}+R_{\mathrm{M}}$. In general this represents a significant improvement as the value $R_{\mathrm{M}}$ can be made very small at an almost negligible cost to transparency - for example, mesoscopic copper wires with $p / w=100$ and $h=1 \mu \mathrm{m}$ will shade just $1 \%$ of the incident radiation at $R_{\mathrm{M}} \approx 2 \Omega \mathrm{sq}^{-1}$. By comparison, a typical TC with comparable sheet resistance would block around $20 \%$ of the incoming light or more. ${ }^{2}$ Within fabrication constraints the wires can be made taller to reduce $R_{\mathrm{M}}$ at no cost to transparency, which is therefore equivalent to reducing the metal's intrinsic resistivity $\rho$. Meanwhile, the factor $(p / W)^{2}$ in the effective sheet resistance can drastically reduce the impact of the TC's sheet resistance $R_{\mathrm{S}}$ in a metallized electrode when the wires are spaced close together, allowing for the use of highly transparent TCs with a larger sheet resistance than would otherwise be optimal. For this purpose the wire width $w$ must be made as small as possible so that the wires do not incur a large shading loss when closely spaced.

The $W^{2}$ scaling of ohmic loss with cell width makes this parameter an important determinant of sheet resistance requirements. In a monolithic thin-film module the tendency to make $W$ smaller in order to reduce ohmic loss is countered by the necessary presence of interconnect regions, which represent undesirable dead space and should therefore be spaced far apart. Technological limits on the width of these interconnect regions set the balance at roughly $W \approx 1 \mathrm{~cm}$, which leads to a required sheet resistance without metallization of about $10 \Omega$ $\mathrm{sq}^{-1}{ }^{9}{ }^{9}$ This well-known figure is visible in Fig. 2 where we have plotted the maximum allowable sheet resistance in order to keep losses under $5 \%$, versus cell width, assuming a bare transparent conductor with perfect transparency (blue dotted line). The same quantity is also plotted for a metallized layer, but this time including the effect of wire shading in the $5 \%$ loss (red solid line). For longer cell widths metallization relaxes the required sheet resistances by a large amount, indeed by over two orders of magnitude at $W=1 \mathrm{~cm}$. Another important point regarding $W$ is that whereas for a bare electrode the cell length affects the voltage loss through $R_{\mathrm{s}}$, in a metallized electrode the variation in loss with length scales with $R_{\mathrm{M}}$, tying the optimal value for $W$ to the wire height. Taller wires with their reduced $R_{\mathrm{M}}$ will allow for larger values of the cell width, which can reduce the negative impact of interconnect dead-space and thereby enhance module efficiency (see ESI $\dagger$ for quantitative estimates).

Just as the ohmic losses discussed above incur a reduction in cell voltage $V_{\mathrm{mp}} / V_{\mathrm{mp}}^{0}$, the effect of non-ideal transparency is to reduce the cell's nominal photocurrent $J_{\mathrm{mp}}^{0}$ by a factor $\left(1-\frac{w}{p}\right)$ from the wire shading, and further by a factor $T$ due to the TC's transparency. At the module level, current is again reduced by a factor $\left(1-\frac{s}{W}\right)$ due to the interconnect space discussed above, where $s$ is the interconnection width. Taken together the ohmic and current losses reduce power generation efficiency by a factor of $J_{\mathrm{mp}} / J_{\mathrm{mp}}^{0} \times V_{\mathrm{mp}} / V_{\mathrm{mp}}^{0}$, or

$$
E\left(R_{\mathrm{s}}, T\right)=\left\{\begin{array}{l}
T\left(1-\frac{s}{W^{*}}\right)\left(1-\frac{1}{3} \frac{J_{\mathrm{mp}}^{0}}{V_{\mathrm{mp}}^{0}} W^{*^{2}} R_{\mathrm{s}}\right), \\
T\left(1-\frac{s}{W^{*}}\right)\left(1-\frac{w}{p^{*}}\right) \times \\
\left(1-\frac{1}{3} \frac{J_{\mathrm{mp}}^{0}}{V_{\mathrm{mp}}^{0}}\left[W^{*^{2}} R_{\mathrm{M}}+\frac{1}{4} p^{*^{2}} R_{\mathrm{s}}\right]\right)
\end{array}\right.
$$

which is defined as a fraction of the theoretical efficiency calculated in the absence of either loss mechanism, again for a bare and metallized TC respectively. The object of designing a transparent electrode is therefore to make this "efficiency factor" as close as possible to unity. The asterisks on $p$ and $W$ denote that the wire spacing and cell width should always be chosen so that $E$ takes its maximum value, conditions which could in principle be used to eliminate these variables from the expressions for $E$. This is straightforward to accomplish by hand in the case of eqn (2a) (see Section 3 below), but less so for eqn (2b), for which we resort to numerical optimization in the calculations to follow. As the transparency $T$ is a common factor in eqn (2), $E$ can be written in the form $E\left(R_{\mathrm{s}}, T\right)=$ $T E_{\max }\left(R_{\mathrm{s}}\right)$ which defines an envelope function $E_{\max }\left(R_{\mathrm{s}}\right)$ denoting the maximum fractional efficiency allowed by 


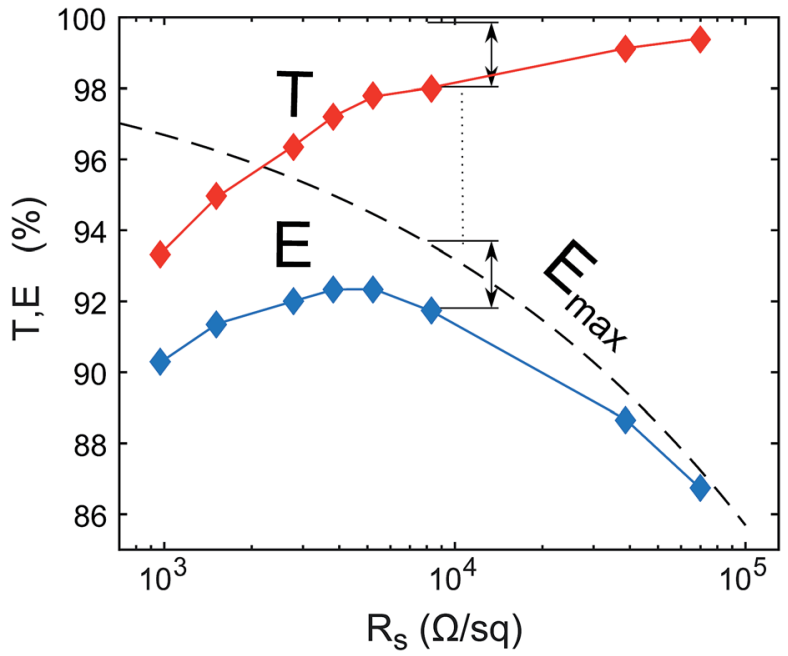

Fig. 3 Experimental transparency and sheet resistance values for the carbon nanotube layers of ref. 28 (red upper series) and the corresponding efficiency factors (blue lower series) after including metallization with $w=30 \mu \mathrm{m}$ and $W=1 \mathrm{~cm}$. Each point lies below the maximal efficiency factor $E_{\max }$ (dotted) by a distance proportional to (1 $-7)$.

current and ohmic losses at a given sheet resistance. The shape of $E_{\mathrm{max}}$ is determined by the parameters $J_{\mathrm{mp}}^{0} / V_{\mathrm{mp}}^{0}$ and $s$, and the metallization parameters $R_{\mathrm{M}}$ and $w$ when they apply. In Fig. 3 we illustrate the role of $E_{\max }$ and demonstrate how the transformation from $\left(R_{\mathrm{s}}, T\right)$ data (red line) to $\left(R_{\mathrm{s}}, E\right)$ data (blue line) unambiguously reveals the optimal TC layer for a specific cell type.
In Fig. 4 we have used published $R_{\mathrm{s}}, T$ data ${ }^{28,30-38}$ to compute efficiency factors for a variety of TC technologies with and without a metal grid. ${ }^{29}$ The cell parameters are taken to correspond roughly with a state-of-the-art perovskite cell, so that $J_{\mathrm{mp}}^{0} / V_{\mathrm{mp}}^{0}=25 \mathrm{~mA} \mathrm{~cm}{ }^{-2} / 1.1 \mathrm{~V}=22 \mathrm{k}^{-1} \mathrm{~cm}^{-2}$, and we use an interconnection width of $s=200 \mu \mathrm{m} .{ }^{9}$ The shaded regions in Fig. 4 denote unphysical efficiency factors defined by $E>E_{\max }$, and each data point is situated at the coordinates $\left(R_{\mathrm{s}}, T E_{\max }\right)$. This comparison reveals that the wire width plays a deciding role in determining which TC technologies give acceptable performance in a solar device. For moderate wire widths of $w=$ $30 \mu \mathrm{m}$ (Fig. 4(a)), corresponding roughly to the capabilities of industrial screen printing, sheet resistances up to a few hundreds of Ohms per square remain competitive, and in that region the competition between technologies is fairly close. For smaller wires of width $w=5 \mu \mathrm{m}$ the playing field is almost completely leveled, and high performance can be obtained with sheet resistances as large as several thousands of Ohms per square (Fig. 4(b)). By contrast, without metallization electrode performance drops off rapidly with increasing sheet resistance and the best candidates lie in the region of 10-50 $\Omega \mathrm{sq}^{-1}$ (Fig. 4(c)). Comparing the best performers in each case we find that a relative efficiency improvement of approximately $5 \%$ can be expected in moving from bare to metallized electrodes. It is also clear that transparency becomes the primary measure of TC quality when smaller wire widths are available. This means that emerging TC technologies such as carbon-nanotubes, which are generally regarded as having unsuitably high sheet resistance for solar applications, can give competitive and even superior performance with the use of narrow-width metallization due to their ultra-high transparency. ${ }^{39}$ Significantly, nearly all of the
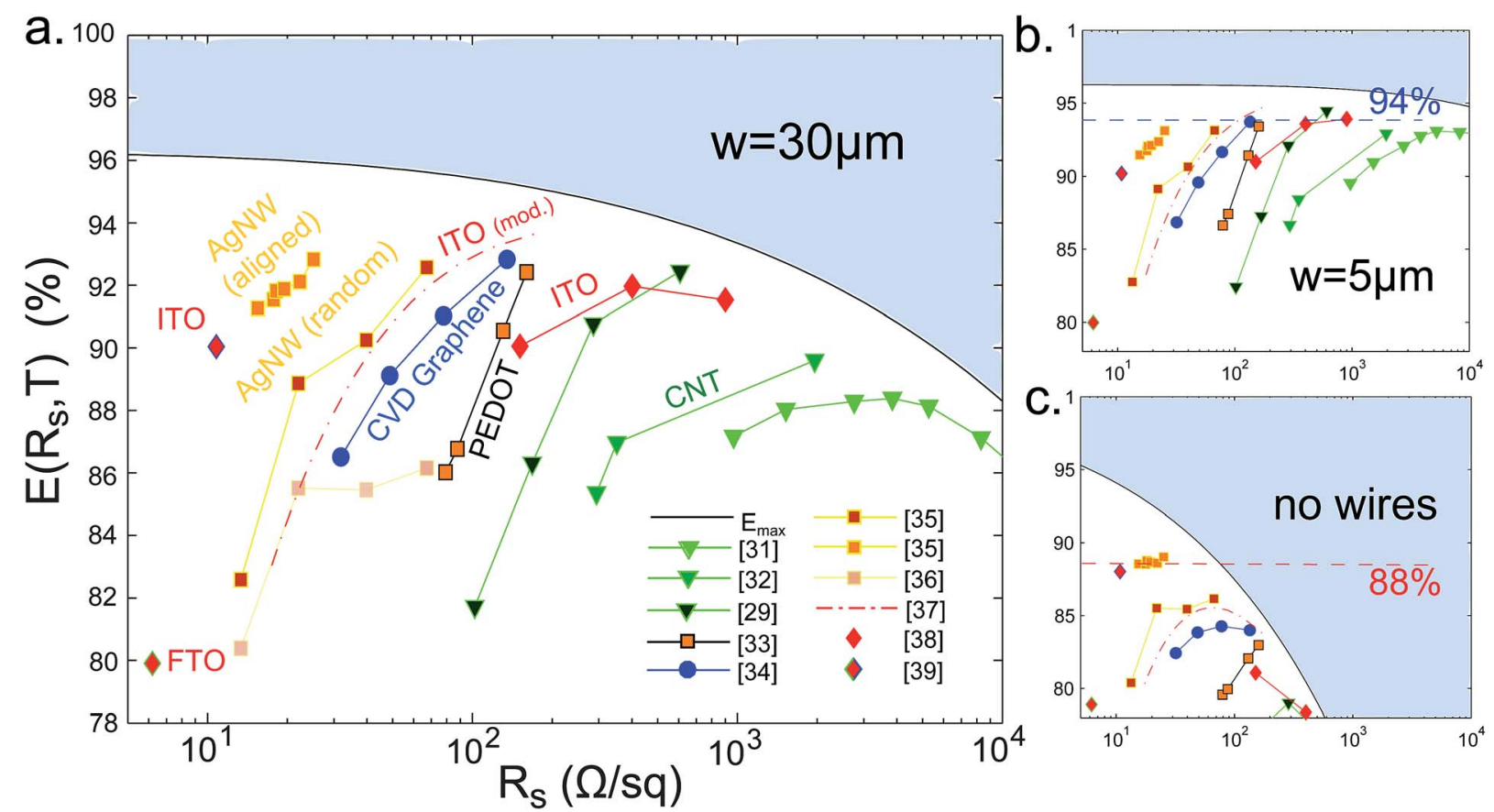

Fig. 4 Plots of the efficiency factor $E$ calculated using experimental $\left(R_{\mathrm{s}}, T\right)$ data from the literature. ${ }^{29}$ The wire widths are $30 \mu \mathrm{m}$ in (a), $5 \mu \mathrm{m}$ in (b), whereas (c) corresponds to a bare electrode. In each case the interconnect width is set to $200 \mu \mathrm{m}$ and for (a) and (b) the wire height is $3 \mu \mathrm{m}$. 
data in Fig. 4(a and b) trends steeply upwards with increasing sheet resistance, suggesting that in most cases the optimal transparency for these technologies (when augmented with a metal grid) has yet to be demonstrated.

The efficiency factors defined in (2) were introduced for the top electrode of a stand-alone cell, but a similar strategy can be pursued to model the performance of TCs in tandem cells with only slightly different equations. We include such an analysis in the ESI. $\dagger$

\section{Figures of merit for solar cells}

To ease comparisons TCs are often ranked in terms of a figure of merit which is a simple function of sheet resistance and transparency. The most widely used is the DC to optical conductivity ratio ${ }^{40}$

$$
\sigma_{\text {d.c. }} / \sigma_{\text {opt }}=\left[\left(T^{-\frac{1}{2}}-1\right) \frac{2 R_{\mathrm{s}}}{Z_{0}}\right]^{-1}
$$

in which $Z_{0}=377 \Omega$. Although this relationship only strictly applies to a thin uniform layer when $\sigma_{\text {d.c. }}$ and $\sigma_{\text {opt }}$ can be separately defined, it can nevertheless be used as an abstract figure of merit for any transparent conductor. An alternative to this is Haacke's function ${ }^{\mathbf{4 1}}$

$$
\Phi_{\mathrm{H}}=T^{q} / R_{\mathrm{s}}
$$

in which $q$ is arbitrary and can be chosen to suit the application, with typical values lying in the range $q=20-100$. The test of a good figure of merit for solar applications is that electrodes with equal figures of merit should give roughly equal performance in a cell. Of course the efficiency factor satisfies this

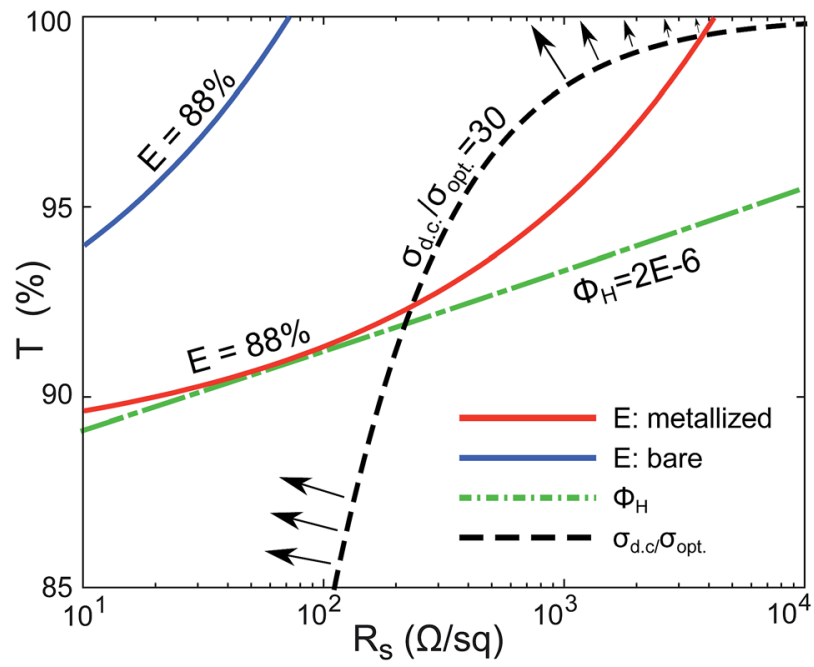

Fig. 5 Level curves of the d.c. to optical conductivity ratio (black dashed) and Haacke's function (green dot-dash), as compared to contours of equal loss factor computed using eqn (5) (blue) and (6) (red). For the bare loss factor we have set $s=200 \mu \mathrm{m}$, the wire width $w$ $=30 \mu \mathrm{m}$ and in both cases $J_{\mathrm{mp}}^{0} / V_{\mathrm{mp}}^{0}=22 \mathrm{k}^{-1} \mathrm{~cm}^{-2}$. Normallyoriented arrows on the conductivity ratio point in the direction of maximum "improvement". criterion by definition within the modelling approximations. To assess the others in Fig. 5 we have plotted level curves of the conductivity ratio and Haacke's function alongside level curves of the efficiency factor for a bare and metallized TC. Although with a hand-picked value for $q$ Haacke's function can be made to follow the efficiency factor approximately (here we have chosen $q=100$ to approximate the metallized curve), the more commonly used conductivity ratio has level curves which can be seen to bend distinctly in the wrong direction. As the normal to each level curve points in the direction of maximum change, the conductivity ratio suggests further decreasing the sheet resistance when it is lowest, and further increasing the transparency when it is already high. This is opposite to the common sense prediction, borne out by the efficiency factor, that these two properties must be balanced in a good electrode with diminishing returns applying as either property is improved. We conclude that the conductivity ratio cannot be relied upon to accurately reflect the performance of a transparent conductor in a solar cell (and indeed, is likely to be similarly unphysical for any application), although Haacke's figure may do a reasonable job if the parameter $q$ is properly chosen.

For a bare electrode eqn (2a) can readily be put in the form of a simple figure of merit as the optimization problem for $W$ has a simple analytic solution. This gives the expression:

$$
\begin{aligned}
& E=T\left(1-\frac{s}{W^{*}}\right)\left(1-\frac{1}{3} \frac{J_{\mathrm{mp}}^{0}}{V_{\mathrm{mp}}^{0}} W^{* 2} R_{\mathrm{s}}\right), \\
& W^{*}=\left(\frac{3 V_{\mathrm{mp}}^{0} s}{2 J_{\mathrm{mp}}^{0} R_{\mathrm{s}}}\right)^{1 / 3}
\end{aligned}
$$

(for further detail see ESI $\dagger$ ). However, with metallization included the problem of obtaining a simple expression for $E_{\max }$ is greatly complicated by the coupling between the optimal wire spacing $p^{*}$ and cell width $W^{*}$. A limiting expression may be obtained by effectively taking the wire height $h \rightarrow \infty$ (or $R_{\mathrm{M}} \rightarrow$ $0)$, giving

$$
\begin{aligned}
& E\left(R_{\mathrm{s}}, T\right)=T\left(1-\frac{w}{p^{*}}\right)\left(1-\frac{1}{12} p^{* 2} \frac{J_{\mathrm{mp}}^{0}}{V_{\mathrm{mp}}^{0}} R_{\mathrm{s}}\right) \\
& p^{*}=\left(\frac{6 V_{\mathrm{mp}}^{0} w}{J_{\mathrm{mp}}^{0} R_{\mathrm{s}}}\right)^{1 / 3}
\end{aligned}
$$

in which the dependence on cell width has completely vanished. Eqn (5) and (6) are both approximate forms of the efficiency factor defined earlier (eqn $(2 a, b))$, but do a good job of capturing the effects of transparent conductor on solar cell performance (see $\mathrm{ESI} \dagger$ for a direct comparison between these figures of merit and the full efficiency factors defined in eqn (2)). Although there appear to be quite a number of parameters in eqn (5) and (6), the cell is completely determined by the characteristic ratio $J_{\mathrm{mp}}^{0} / V_{\mathrm{mp}}^{0}$ and interconnect width $s$ in the unmetallized case, and in the metallized case by $J_{\mathrm{mp}}^{0} / V_{\mathrm{mp}}^{0}$ together with the wire width $w$. Typically $J_{\mathrm{mp}}^{0} / V_{\mathrm{mp}}^{0}$ lies in the range of 20$50 \mathrm{k} \Omega^{-1} \mathrm{~cm}^{-2}$ depending on the cell type, ${ }^{\mathbf{4 2 , 4 3}}$ the width $s$ lies in the range of $100-1000 \mu \mathrm{m},{ }^{9}$ and $w=30 \mu \mathrm{m}$ corresponds roughly to the current capabilities of industrial screen printing. ${ }^{15}$ These 
expressions therefore represent simple alternatives to the standard TC figures of merit with a direct correspondence to actual solar cell performance.

\section{Conclusions}

Metallization relieves the burden on a transparent conductor's sheet resistance, leveling the competition between technologies and allowing for other factors such as material compatibility or ease of processing to take precedence. Whenever it is possible to incorporate a narrow metallic grid $(w<30 \mu \mathrm{m})$, the requirements for a transparent conductor shift dramatically towards achieving ultra-high transparency (>95\%). Wire widths in the range of $20-30 \mu \mathrm{m}$ already relax the conventional sheet resistance requirement of $10 \Omega \mathrm{sq}^{-1}$ by more than an order of magnitude, encompassing a wide range of emerging technologies, whilst widths approaching $5 \mu \mathrm{m}$ will allow for several thousands of ohms per square in the transparent layer. Furthermore, we have shown that metallized transparent layers generally exceed the performance of the best bare transparent conductors due to a combined reduction of losses at the cell and module level. Narrow-width metallization therefore provides a clear route for approaching ideal electrode performance in thin-film solar cells. These findings demonstrate the potential for scalable methods of incorporating thin metal wires into cell substrates to disrupt the transparent conductor landscape.

For sensitive cell architectures embedding of the wires may be necessary to avoid drastic nonuniformities, and this is likely to be simpler for flexible polymer substrates than for glass. However even in the latter case one may look to the literature of microfluidics where the problem of structuring glass has received considerable attention, ${ }^{\mathbf{4 4 , 4 5}}$ or to well-established solgel techniques ${ }^{12,13}$ for imprinting patterns onto glass. Once embedded, these metal wires could be made very much taller than otherwise in order to accommodate larger cell widths, providing a reduction in module dead-space. Electrodes which are naturally exposed, such as the rear electrode in a perovskite four-terminal tandem device, can be easily metallized and researchers should be aware that this obviates the standard requirement of $10 \Omega \mathrm{sq}^{-1}$.

As a figure of merit for solar cells we have shown that the widely used optical to d.c. conductivity ratio exhibits highly unphysical biases in the low and high $R_{\mathrm{s}}$ regimes, and particularly overestimates the value of having low sheet resistance, even for un-metallized electrodes. By modeling their expected impact on solar cell efficiency, we have suggested new figures of merit for transparent conductors with clear relevance for solar applications and a sound physical basis.

\section{Acknowledgements}

The authors gratefully acknowledge The Duong for his contribution of the TCO film data appearing in Fig. 4, and Niraj Lal for useful discussions. KC acknowledges support from the Australian Research Council Future Fellowship Scheme. This work has been supported by the Australian Government through the Australian Renewable Energy Agency (ARENA). Responsibility for the views, information or advice expressed herein is not accepted by the Australian Government.

\section{References}

1 D. S. Hecht, L. Hu and G. Irvin, Adv. Mater., 2011, 23, 14821513.

2 K. Ellmer, Nat. Photonics, 2012, 6, 809-817.

3 M. Layani, A. Kamyshny and S. Magdassi, Nanoscale, 2014, 6, 5581-5591.

4 H. J. Snaith, J. Phys. Chem. Lett., 2013, 4, 3623-3630.

5 M. A. Green, A. Ho-Baillie and H. J. Snaith, Nat. Photonics, 2014, 8, 506-514.

6 N. Lal, T. White and K. Catchpole, Journal of Photovoltaics, 2014, 4, 1380-1386.

7 C. D. Bailie, M. G. Christoforo, J. P. Mailoa, A. R. Bowring, E. L. Unger, W. H. Nguyen, J. Burschka, N. Pellet, J. Z. Lee, M. Gratzel, R. Noufi, T. Buonassisi, A. Salleo and M. D. McGehee, Energy Environ. Sci., 2015, 8, 956-963.

8 P. Löper, S.-J. Moon, S. Martín de Nicolas, B. Niesen, M. Ledinsky, S. Nicolay, J. Bailat, J.-H. Yum, S. De Wolf and C. Ballif, Phys. Chem. Chem. Phys., 2015, 17, 1619-1629.

9 M. W. Rowell and M. D. McGehee, Energy Environ. Sci., 2011, 4, 131-134.

10 J. Wennerberg, J. Kessler and L. Stolt, Sol. Energy Mater. Sol. Cells, 2001, 67, 59-65.

11 T. Duong, N. Lal, D. Grant, D. Jacobs, P. Zheng, S. Rahman, H. Shen, M. Stocks, A. Blakers, K. Weber, T. P. White and K. R. Catchpole, IEEE Journal of Photovoltaics, 2016, PP, 1-9. 12 C. Marzolin, S. P. Smith, M. Prentiss and G. M. Whitesides, Adv. Mater., 1998, 10, 571-574.

13 O. J. A. Schueller, G. M. Whitesides, J. A. Rogers, M. Meier and A. Dodabalapur, Appl. Opt., 1999, 38, 5799-5802.

14 K. Tvingstedt and O. Inganäs, Adv. Mater., 2007, 19, 2893-2897. 15 M. Ju, Y.-J. Lee, J. Lee, B. Kim, K. Ryu, K. Choi, K. Song, K. Lee, C. Han, Y. Jo and J. Yi, Sol. Energy Mater. Sol. Cells, 2012, 100, 204-208.

16 H. Sirringhaus, T. Kawase, R. H. Friend, T. Shimoda, M. Inbasekaran, W. Wu and E. P. Woo, Science, 2000, 290, 2123-2126.

17 Z. Li, P.-C. Hsiao, W. Zhang, R. Chen, Y. Yao, P. Papet and A. Lennon, Energy Procedia, 2015, 67, 76-83.

18 A. Lorenz, A. Senne, J. Rohde, S. Kroh, M. Wittenberg, K. Kruger, F. Clement and D. Biro, Energy Procedia, 2015, 67, 126-137.

19 S. Thibert, D. Chaussy, D. Beneventi, N. Reverdy-Bruas, J. Jourdan, B. Bechevet and S. Mialon, 38th Photovoltaic Specialists Conference, 2012, pp.2266-2270.

20 S. H. Ahn and L. J. Guo, Adv. Mater., 2008, 20, 2044-2049.

21 A. Kumar and G. M. Whitesides, Appl. Phys. Lett., 1993, 63, 2002-2004.

22 Y.-L. Loo, R. L. Willett, K. W. Baldwin and J. A. Rogers, Appl. Phys. Lett., 2002, 81, 562-564.

23 P.-C. Hsu, S. Wang, H. Wu, V. K. Narasimhan, D. Kong, H. Ryoung Lee and Y. Cui, Nat. Commun., 2013, 4, 2522.

24 J. Zou, H.-L. Yip, S. K. Hau and A. K.-Y. Jen, Appl. Phys. Lett., 2010, 96, 203301. 
25 Y. Zhu, Z. Sun, Z. Yan, Z. Jin and J. M. Tour, ACS Nano, 2011, 5, 6472-6479.

26 G. T. Koishiyev and J. R. Sites, Sol. Energy Mater. Sol. Cells, 2009, 93, 350-354.

27 M. Green, Centre Photovoltaic Devices \& Systems, Silicon Solar Cells: Advanced Principles \& Practice, 1995, p. 366.

28 H.-Z. Geng, K. K. Kim, K. P. So, Y. S. Lee, Y. Chang and Y. H. Lee, J. Am. Chem. Soc., 2007, 129, 7758-7759.

29 In all figures we have used transparency values reported at $550 \mathrm{~nm}$, with the exception of the transparent oxide films (ITO and FTO) which generally exhibit a considerable variation in transmission with wavelength. For ITO and FTO each transmittance was weighted with the AM1.5g spectrum. For the other technologies full spectral data was not available.

30 V. Scardaci, R. Coull and J. N. Coleman, Appl. Phys. Lett., 2010, 97, 5-8.

31 J. Li, L. Hu, L. Wang, Y. Zhou, G. Grüner and T. J. Marks, Nano Lett., 2006, 6, 2472-2477.

32 J. E. McCarthy, C. a. Hanley, L. J. Brennan, V. G. Lambertini and Y. K. Gun'ko, J. Mater. Chem. C, 2014, 2, 764-770.

33 S. Bae, H. Kim, Y. Lee, X. Xu, J.-S. Park, Y. Zheng, J. Balakrishnan, T. Lei, H. R. Kim, Y. I. Song, Y.-J. Kim, K. S. Kim, B. Ozyilmaz, J.-H. Ahn, B. H. Hong and S. Iijima, Nat. Nanotechnol., 2010, 5, 574-578.

34 S. Kang, T. Kim, S. Cho, Y. Lee, A. Choe, B. Walker, S.-J. Ko, J. Y. Kim and H. Ko, Nano Lett., 2015, 15, 7933-7942.

35 Silver nanowire data ${ }^{34}$ (random array) with the efficiency factor calculated without metallization (i.e. for a bare electrode), included for comparison.

36 For this series we have modelled the transmission through ITO layers of varying thickness using the Beer-Lambert formula for absorption, taking the extinction coefficient from ref. 46 and neglecting reflection.

37 In-house measurements of RF-sputtered ITO deposited at room temperature (see ref. 11 for further details).

38 In-house measurements of commercial samples of ITO (Sigma Aldrich $578274, t \approx 120 \mathrm{~nm}$ on glass) and FTO (Dyesol TEC7, $t \approx 650 \mathrm{~nm}$ on glass). As the reflectance of these thin-films can vary widely we have elected to include only absorption in the transparency data, so that $T=1-A$, making these values an upper estimate.

39 We note that a significant loss in voltage or collection efficiency may result if the mobility of the underlying active layer is not sufficient to ensure a smooth passage for carriers to the individual tubes (for CNT layers) or nanowires (AgNW electrodes), an issue which may arise in certain organic semiconductors.

40 S. De and J. N. Coleman, MRS Bull., 2011, 36, 774-781.

41 G. Haacke, J. Appl. Phys., 1976, 47, 4086-4089.

42 K. Ramanathan, J. Keane and R. Noufi, Conference Record of the Thirty-first IEEE Photovoltaic Specialists Conference, 2005, pp. 195-198.

43 X. Wu, R. G. Dhere, D. S. Albin, T. A. Gessert, C. DeHart, J. C. Keane, A. Duda, T. J. Coutts, S. Asher, D. H. Levi and et al., in High-Efficiency CTO/ZTO/CdS/CdTe Polycrystalline Thin-Film Solar Cells: Preprint, 2001.

44 M. Stjernström and J. Roeraade, J. Micromech. Microeng., 1999, 8, 33-38.

45 C. Iliescu, F. E. H. Tay and J. Miao, Sens. Actuators, A, 2007, 133, 395-400.

46 Z. C. Holman, M. Filipič, A. Descoeudres, S. DeWolf, F. Smole, M. Topič and C. Ballif, J. Appl. Phys., 2013, 113, 013107. 